\title{
Fertility Preservation Indications and Available Options for Adult Non-Cancer Individuals
}

\author{
Murid Javed* and Hamad Al Sufyan \\ Thuriah Medical Center, Saudi Arabia \\ *Corresponding author: Murid Javed, Thuriah Medical Center, Saudi Arabia
}

\begin{tabular}{l}
\hline ARTICLE INFO \\
\hline Received: 幽 August 13, 2019 \\
Published: 业 August 26, 2019 \\
\hline Citation: Murid Javed, Hamad Al Sufyan. \\
Fertility Preservation Indications and \\
Available Options for Adult Non-Cancer \\
Individuals. Biomed J Sci \& Tech Res \\
20(5)-2019. BJSTR. MS.ID.003514.
\end{tabular}

\begin{abstract}
Fertility preservation has been traditionally recommended for cancer and infertile patients. However, it is now increasingly utilized by healthy men and women due to personal preferences or demands of the society. For women, oocyte and embryo cryopreservation is highly successful. Ovarian tissue cryopreservation and re-implantation, though successfully utilized, is still experimental. For men, sperm retrieved from ejaculate or surgical intervention of epididymis and testes have long been successfully cryopreserved. Outcome of fresh or cryopreserved sperm are similar. Indication of fertility preservation varies depending on medical or genetic condition, personal preference, career requirement or apprehensible risk of damage to gametes. This mini- review highlights multiple indications and available options for fertility preservation in non-cancer adult men and women.
\end{abstract}

Keywords: Fertility Preservation; Oocytes; Embryos; Ovarian Tissue; Testicular Tissue; Vitrification; Menopause; Infertility

\section{Introduction}

Human society has changed significantly due to industrial and information technology revolution. Lifestyle changes, personal preferences, society demands, increased cost of family raising, dietary patterns, increased use of insecticides and pesticides, increased use of electronics, career competition and ideological changes have affected human fertility and family structure. The environmental changes have negatively impacted the reproductive health, the effects of which extend beyond the individual and family to the society and even to the World [1,2]. These effects are more pronounced in women. Although men can often maintain fertility potential for lifetime, however, the evidence indicates worsening of semen parameters, including sperm genetics, and reduced reproductive success [3]. Improvements in oocyte, sperm, embryo, ovarian and testicular tissue cryopreservation in association with advancements in endocrinology and surgery have opened new avenues. Men with reproductive problems like disorders of sex development, cryptorchidism, hypospadias, low testosterone levels and poor semen quality [4] can preserve their fertility. There is a great need for fertility preservation counseling before initiation of any treatment that may affect the gametes production and quality. The objectives of this mini- review are to highlight indications and currently available fertility preservation options for adult noncancer individuals.

\section{Fertility Preservation Indications for Women}

The demand of fertility preservation by non-oncological adult women has increased tremendously in recent years. The indications are given below.

Delaying Childbearing: The women are increasingly delaying childbearing due to career competition, employment requirement, non-availability of suitable partner or other personal reasons. To avoid oocyte depletion, oocyte cryopreservation is an alternate to have biological children in future. In the United States, female obstetrics and gynecology residents are increasingly choosing to preserve their oocytes due to career and social support reasons [5]. A few prominent companies are offering to cover cost of oocyte cryopreservation to their female employees affecting the trend of fertility preservation. Though fertility preservation may allow a woman opportunity to have her biological children later during her life, however, sufficient information on the safety, efficiency, emotional risks and cost effectiveness is not available. Elective oocyte vitrification in mid-late thirties resulted in good 
outcome after these women utilized IVF in their early forties [6]. The minimum number of mature oocytes needed to achieve a reasonable outcome is estimated to be 8-10 in women aged about 35 years. More oocytes are needed as the age advances. Women choosing this option should cryopreserve their oocytes at younger age to increase possibility of success [7].

Delaying Menopause: A few days ago, in the United Kingdom, ovarian tissue cryopreservation, warming and re-implantation has been used to delay menopause. The idea is to remove a part of the human ovary, cryopreserve it and re-implant back in the same woman before menopause [8]. It is estimated that menopause can be delayed up to 20 years. The cryopreservation of part of the ovary is recommended for women below 40 years of age when their ovaries still have large number of follicles in the ovarian cortex.

Medical Conditions: The list of diseases is increasing in which either the disease or the treatment causes gonadal damage. The success of fertility preservation depends on age of patient, presence or absence of ovarian involvement, available time and the indication of fertility preservation. The most common medical conditions requiring fertility preservation are autoimmune diseases, hematopoietic stem cell transplantation and conditions causing premature ovarian failure [9]. The autoimmune diseases include inflammatory bowel disease, systemic lupus erythematosus, rheumatoid arthritis, pemphigus vulgaris, granulomatosis with polyangiitis, eosinophilic granulomatosis, Bechet's disease or any other autoimmune disorder not responding to immunosuppressive treatment. The blood disorders include sickle cell anemia, plastic anemia and thalassemia major. The premature ovarian failure can be caused by disorders of hypothalamic-pituitary-gonadal axis, oophoritis, benign ovarian tumors, Turner syndrome, fragile $\mathrm{X}$, beta thalassemia, endometriosis and galactosemia [10]. A mutation of germline transcription factor (GATA2) involved in hematopoiesis and angiogenesis was discovered in 2011. Due to the risk of ovarian failure, affected patients should be recommended fertility preservation [11].

Surgery of the Pelvic Area: Surgery in the pelvic area may damage the ovaries or may interfere with its normal physiological function [12]. The bilateral ovarian endometriosis surgeries have a low but definite risk of premature ovarian failure occurring immediately after surgery [13].

Other Conditions: The women with poor ovarian reserve and Turner syndrome can opt for oocyte cryopreservation. Multiple attempts may be needed to get enough number of mature oocytes to create good quality embryos. Depending on the age, 15-20 mature oocytes for cryopreservation are estimated to achieve a live birth [14].

\section{Fertility Preservation Options for Women}

The currently available fertility preservation options for women are preserving mature oocytes, embryos and ovarian tissue [15]. The vitrification is method of choice for oocytes and embryos cryopreservation. The programmable slow cryopreservation and vitrification produce similar pregnancy rates for ovarian tissue cryopreservation.

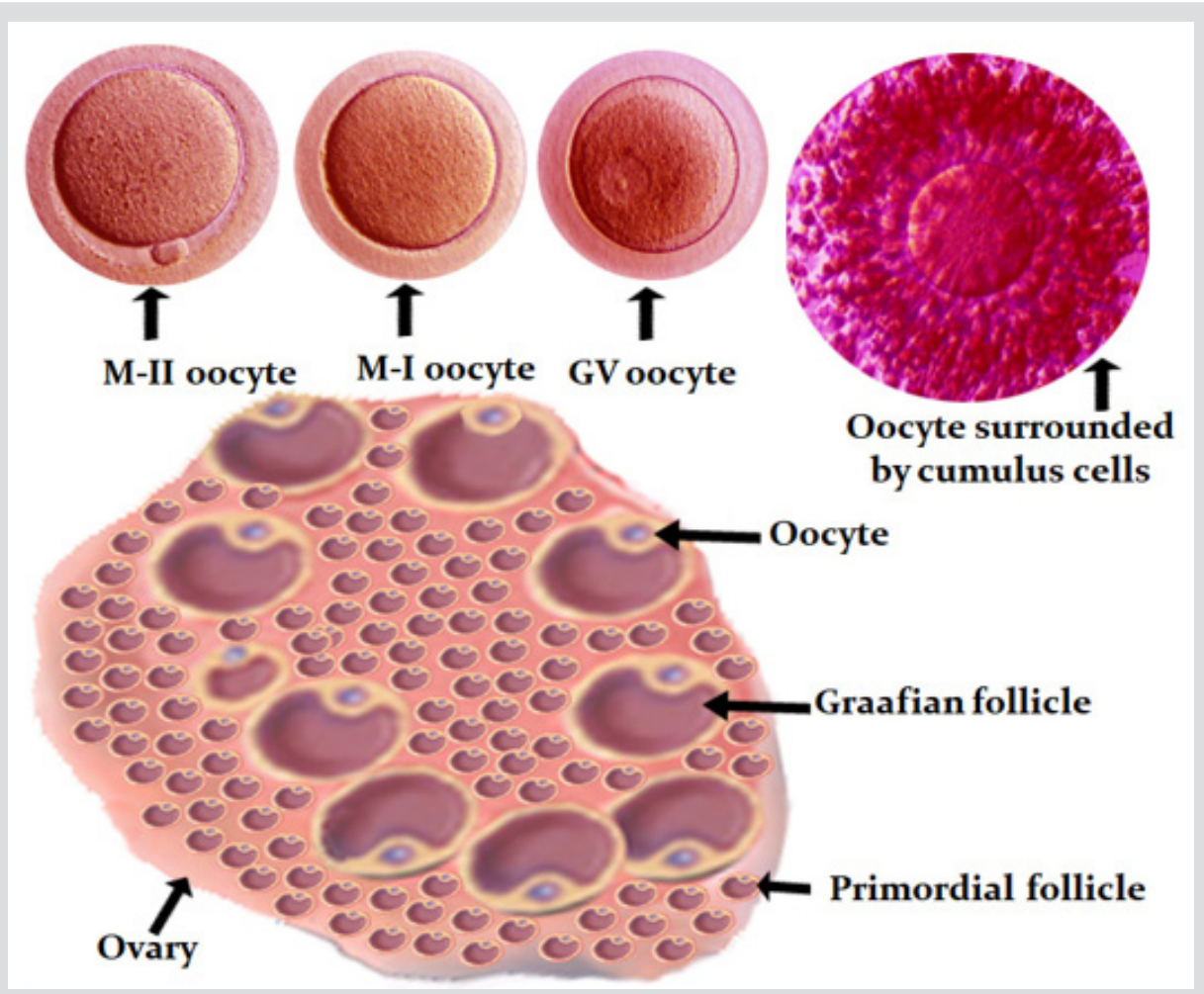

Figure 1: Diagrammatic presentation of human ovary (source of oocytes). In the top row are developmental stages of oocyte and an oocyte surrounded by cumulus cells. The oocyte at MII stage is desired for fertility cryopreservation. 
Oocyte Cryopreservation: In vivo matured oocyte cryopreservation is the only choice for unmarried women. The married women by choice or due to non-availability of sperm on the day of ICSI can cryopreserve oocytes. In advanced age women with poor ovarian reserve, oocyte cryopreservation can be used to pool eggs either for routine IVF/ICSI or for preimplantation genetic testing. Figure 1 shows oocyte source and stages of development. Currently the mature oocytes (MII) survive well and result in live birth. The survival of immature oocytes (GV and MI) is not satisfactory and further studied are needed to improve their outcome [16]. Implantation and clinical pregnancy rates are similar after use of fresh or cryopreserved-warmed mature oocytes [17].
Ovarian tissue cryopreservation: Freezing ovarian tissue or whole ovary are still experimental options. If the ovaries have to be removed, either strips of the ovarian cortex or the whole ovary can be frozen. The cryopreservation of ovarian cortical tissue presents an efficient and high-yield method of potentially preserving thousands of ovarian follicles through a single procedure. Live births have been reported after re-implantation of ovarian strips [18]. Pregnancy rates after re-implantation of ovarian tissue are similar between slow freezing and fast freezing (vitrification) methods; however, vitrified transplants have longer duration of function [19] (Figure 2).

\section{Embryo Development stages that can possibly be cryopreserved}
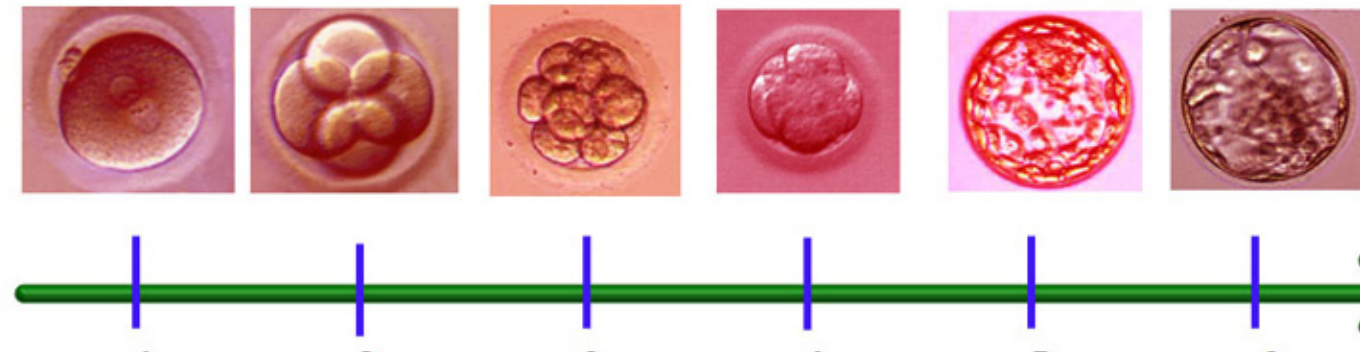

1
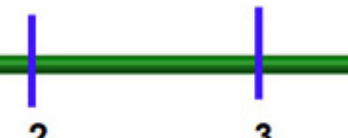

3

4

Days of Embryo Development

Figure 2: Different days and developmental stages at which the embryos can be preserved. After day-7 even if the embryo becomes blastocyst, chances of establishing pregnancy are extremely low.

Embryo Cryopreservation: Embryo cryopreservation is an established method of fertility preservation for married women. Pregnancy and live birth rate after frozen embryo transfer are either equivalent or higher than those achieved after fresh embryo transfer [20]. The embryos have been successfully frozen at different stages of development (Figure 3). Both slow (programmable) and fast (vitrification) procedures have been successfully used. Due to higher survival rate and time saving, vitrification is now almost an exclusive procedure for embryo cryopreservation.

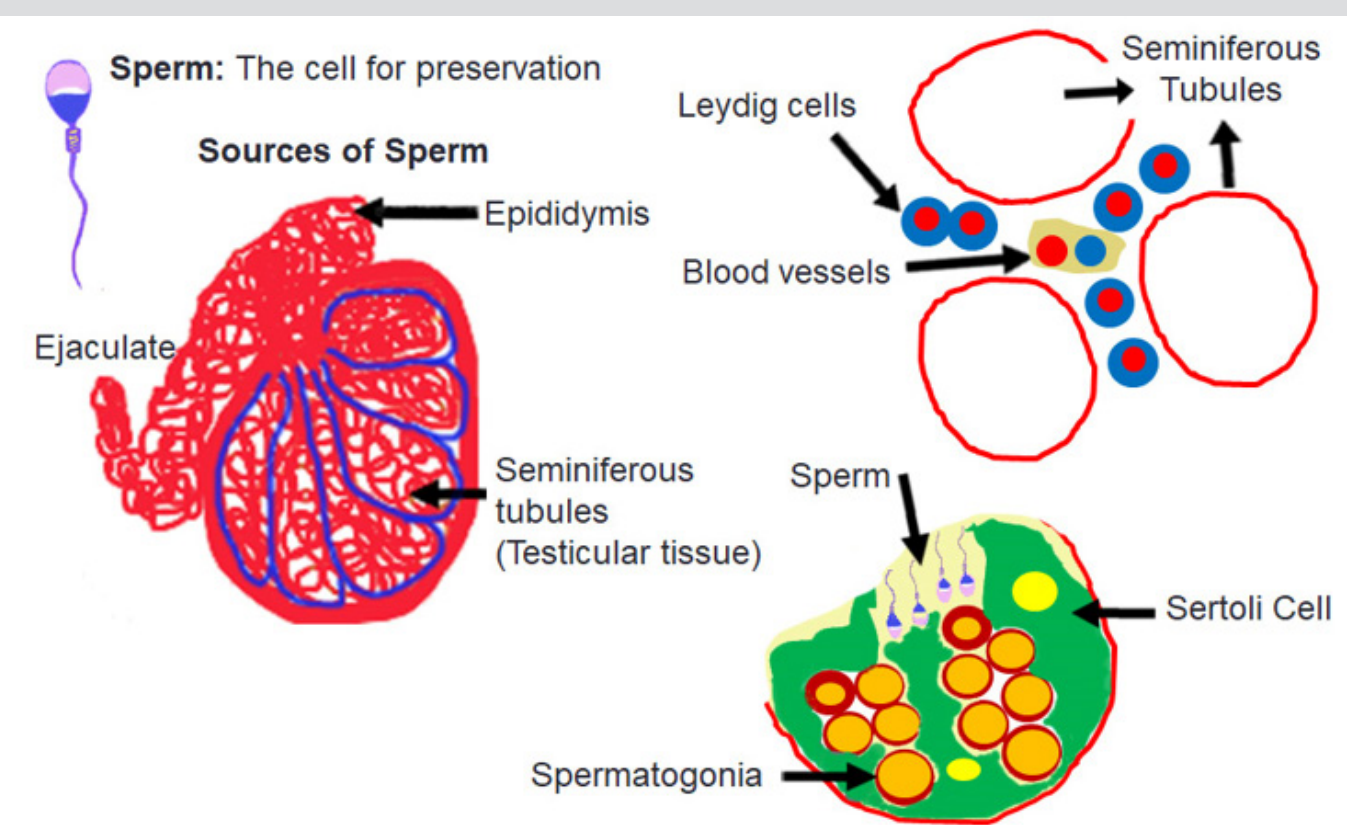

Figure 3: Diagrammatic presentation of human testis and seminiferous tubules. The sperm are made in the seminiferous tubules containing Sertoli and Leydig cells necessary for spermatogenesis. 


\section{Fertility Preservation Indications for Men}

Sperm cryopreservation is an established procedure for fertility preservation. In men with poor semen quality and those who may not be available at the time of assisted reproduction procedure of their partner, semen cryopreservation is recommended. Surgical sperm collection from testicle may not coincide with oocyte aspiration day, or there may be minimal chances of finding sperm by surgical retrieval, therefore, surgical sperm retrieval and cryopreservation are recommended before initiation of ovarian stimulation [21].

Medical Conditions: The medical conditions include systemic lupus erythematosus, multiple sclerosis, diabetes mellitus and cytotoxic therapy for non-cancer conditions like glomerulonephritis [22].

Genetic Condition: The Klinefelter's syndrome is a genetic condition causing azoospermia in about $90 \%$ affected cases [21]. Some affected individuals produce very rare motile sperm. These individuals can be offered to ejaculate on multiple occasions and preserve if motile sperm are found. Alternatively, testicular tissue can be obtained via TESE or Micro-TESE. Such sperm are usable by intracytoplasmic sperm injection. Births have been reported from individuals affected by Klinefelter's syndrome [23].

Other Conditions: For men, who have not yet completed their family and are at risk of Andropause, sperm cryopreservation at an earlier age is a feasible option to safeguard fertility [24]. Testicular tissue cryopreservation at an earlier age and re-implantation at andropause has not been experimented yet. A few other conditions, like severe testicular injury, extensive surgery in the pelvic area and gender reassignment procedures may need testicular tissue or ejaculated sperm cryopreservation. The gender reassignment will make the individual temporarily or permanently infertile. Therefore, in such procedures, enough sperm should be collected via ejaculation or testicular tissue and preserved for future use before initiating hormonal or surgical interventions [20]. Similarly, injury to the spinal cord, surgery of the retroperitoneal lymph node, aortic-iliac reconstruction and colorectal incisions may cause anejaculation. Semen cryopreservation after ejaculation can be repeated after 2-3 days [25].

\section{Hypothalamic-Pituitary-Gonadal Axis Dysfunction}

Men suffering from hypothalamic-pituitary-gonadal axis dysfunction are able to produce sperm at restoration of hormones to physiological level [22]. Such individuals may lose sperm production on discontinuation of therapy. Sperm cryopreservation for such individuals is highly recommended.

\section{Fertility Preservation Options for Men}

The most successful option for men is cryopreservation of ejaculated sperm. Based on the quality of sample, it can later be used for intrauterine insemination, in vitro egg insemination or intracytoplasmic sperm injection. Multiple ejaculates can be combined to provide appropriate number of motile sperm for any of the abovementioned procedure. The cryopreserved samples have been successfully used after 40 years in storage [26].

\section{Conclusion}

Fertility preservation has become a need of adult women and men in changing human society. Significant advances have been made in fertility preservation technologies and results are comparable after use of fresh or cryopreserved gametes. Use of cryopreserved gametes has become an integral part of assisted reproduction in the treatment of infertile couples. Fertility preservation counseling before initiation of any therapy that may damage gametes is essential.

\section{References}

1. Etuk SJ (2009) Keynote Address-Reproductive health global infertility trend. Nigerian Journal of Physiological Sciences 24(2).

2. Mitra S, Murmu N, Rahman SM, Nandi P (2018) Environmental Effect on Male Infertility Preventive Therapeutic Approach. Practical Guide in Andrology and Embryology 30: 117.

3. Mazur DJ, Lipshultz LI (2018) Infertility in the aging male Current urology reports. 19(7): 54.

4. Skakkebaek NE, Rajpert-De ME, Buck LGM, Toppari J, Andersson AM, et al. (2016) Male Reproductive Disorders and Fertility Trends Influences of Environment and Genetic Susceptibility. Physiol Rev 96(1): 55-97.

5. Esfandiari N, Litzky J, Sayler J, Zagadailov P, George K, et al. (2019) Egg freezing for fertility preservation and family planning a nationwide survey of US Obstetrics and Gynecology residents. Reproductive Biology and Endocrinology 17(1): 16.

6. Schlenker TW, McCormick S, Smith R, Pospisil C, Schoolcraft WB, et al. (2018) Efficiency of elective oocyte vitrification for reproductive aging women seeking fertility preservation to delay motherhood. Fertility and Sterility 110(4): e187.

7. Cobo A, García-Velasco JA, Coello A, Domingo J, Pellicer A, et al. (2016) Oocyte vitrification as an efficient option for elective fertility preservation. Fertility and sterility 105(3): 755-764.

8. Swerling G (2019) Menopause breakthrough will allow older women to have children after building a career, doctor claims.

9. Sonmezer M, Oktay K (2004) Fertility preservation in female patients, Human Reproduction Update 10(3): 251-266.

10. Martinez F, Andersen CY, Barri PN, Brannigan R, Cobo A, et al. (2017) Update on fertility preservation from the Barcelona International Society for Fertility Preservation-ESHRE-ASRM 2015 expert meeting: indications, results and future perspectives. Fertility and sterility 108(3): 407-415.

11. Zolton JR, Parikh T, Pilgrim J, Hickstein DD, Holland SM, et al. (2017) Fertility preservation for GATA2 patients. Fertility and Sterility 108(3): e183.

12. Roman H, Sentilhes L, Cingotti M, Verspyck E, Marpeau L (2005) Uterine devascularization and subsequent major intrauterine synechiae and ovarian failure. Fertility and sterility 83(3): 755-757.

13. Busacca M, Riparini J, Somigliana E, Oggioni G, Izzo S, et al. (2006) Postsurgical ovarian failure after laparoscopic excision of bilateral endometriomas. American Journal of Obstetrics and Gynecology 195(2): 421-5.

14. Borini A, Coticchio G (2019) Oocyte quantity and quality are crucial for a perspective of fertility preservation in women with Turner syndrome. Fertility and sterility 111(3): 461-462.

15. Donnez J, Dolmans MM (2017) Fertility preservation in women. New England Journal of Medicine 377(17): 1657-1665. 
16. Son WY, Henderson S, Cohen Y, Dahan M, Buckett W (2019) Immature oocyte for fertility preservation. Frontiers in endocrinology 10: 464

17. Pavone ME, Confino R, Steinberg M (2016) Female fertility preservation a clinical perspective. Minerva Ginecologica 68(4): 458.

18. Silber S (2016) Ovarian tissue cryopreservation and transplantation scientific implications. J Assist Reprod Genet 33(12): 1595-1603.

19. Silber SJ, Derosa MJ, Pineda J (2017) Long term results with vitrification compared to slow freeze of ovarian tissue. Fertility and Sterility 108(3): e183.

20. Pontré JC, Ryan JP, Tan A, Hart RJ (2019) The interval transfer of a frozen-thawed embryo is more successful than a fresh embryo transfer for women undergoing IVF with recurrent implantation failure after cleavage stage embryo biopsy. Australian and New Zealand Journal of Obstetrics and Gynaecology 59(1): 134-9.

21. Rozati H, Handley T, Jayasena CN (2017) Process and Pitfalls of Sperm Cryopreservation. J Clin Med 6(9): 89.

ISSN: 2574-1241

DOI: 10.26717/BJSTR.2019.20.003514

Murid Javed. Biomed J Sci \& Tech Res

cC (i) This work is licensed under Creative

Submission Link: https://biomedres.us/submit-manuscript.php
22. Mortimer CH, McNeilly AS, Fisher RA, Murray MA, Besser GM (1974) Gonadotrophin-releasing hormone therapy in hypogonadal males with hypothalamic or pituitary dysfunction. Br Med J 4(5945): 617-621.

23. Palermo GD, Schlegel PN, Sills ES, Veeck LL, Zaninovic N, et al. (1998) Births after intracytoplasmic injection of sperm obtained by testicular extraction from men with nonmosaic Klinefelter's syndrome. New England Journal of Medicine 338(9): 588-590.

24. Roychoudhury S, Bhattacharjee R (2018) Environmental Issues Resulting in Andropause and Hypogonadism. In Bioenvironmental Issues Affecting Men's Reproductive and Sexual Health pp. 261-273. Academic Press.

25. Ayad BM, Van der Horst G, Du Plessis SS (2018) Revisiting the relationship between the ejaculatory abstinence period and semen characteristics. International journal of fertility \& sterility 11(4): 238.

26. Szell AZ, Bierbaum RC, Hazelrigg WB, Chetkowski RJ (2013) Live births from frozen human semen stored for 40 years. Journal of assisted reproduction and genetics 30(6): 743-744.

$\begin{array}{ll}\text { BIOMEDICAL } & \text { Assets of Publishing with us } \\ \text { RESEARCHES } & \text { - Global archiving of articles } \\ & \text { - Immediate, unrestricted online access } \\ & \text { - Rigorous Peer Review Process } \\ \end{array}$

